\title{
ECOLOGICAL
} MODELLING

Ecological Modelling 159 (2003) 133-143

www.elsevier.com/locate/ecolmodel

\section{Apparent differences in stochastic growth rates based on timing of census: a cautionary note}

\author{
Evan G. Cooch ${ }^{\mathrm{a}, *}$, Gilles Gauthier ${ }^{\mathrm{b}}$, Robert F. Rockwell ${ }^{\mathrm{c}}$ \\ a Department of Natural Resources, Cornell University, Ithaca, NY 14853-3001, USA \\ ${ }^{\mathrm{b}}$ Département de biologie, Centre d'études nordiques, Université Laval, Ste-Foy, Quebec, Canada G1K 7P4 \\ ${ }^{\mathrm{c}}$ Department of Ornithology, American Museum of Natural History, New York, NY 10024-5192, USA
}

Received 7 December 2001; received in revised form 11 July 2002; accepted 26 July 2002

\begin{abstract}
Matrix population models are increasingly used in both theoretical and applied analysis of population dynamics (Caswell, Matrix Population Models-Construction, Analysis, and Interpretation (2001) 722). The projected asymptotic growth rate from deterministic models, based on a single matrix, is invariant as to when in the annual cycle the population is censused (typically, either immediately prior to or after breeding). Thus, we expect that given a set of $n$ matrices $\left\{\mathbf{A}_{1}, \mathbf{A}_{2}, \ldots, \mathbf{A}_{n}\right\}$, where each matrix $\mathbf{A}_{i}$ corresponds to a given environmental state $i$, that the longterm expected stochastic growth rate calculated by the product of matrices selected at random from this set should not depend on whether the matrices in the set are configured based on either a pre- or post-breeding census. However, differences in stochastic growth rate as a function of the timing of the census can arise under conditions where there is significant covariance among the individual matrix elements. Using a seasonal (periodic) matrix modeling approach, we show that such differences are an artifact of how stochasticity is entered into the matrices, particularly when the matrices are structured based on a post-breeding census model. In such cases, the annual projection matrix for a given year is in fact a product of component seasonal matrices from two successive years. Failing to account for this when there is covariance among seasons will lead to a disparity in estimated stochastic growth rate when growth is calculated based on the product of random matrices, as is frequently done. We show that a seasonal matrix approach, where the seasonal matrices are explicitly subscripted for the appropriate year, eliminates the problem, and is a generally robust approach to stochastic modeling.
\end{abstract}

(C) 2002 Elsevier Science B.V. All rights reserved.

Keywords: Matrix models; Population growth; Stochastic growth rate; Timing of census

\section{Introduction}

Matrix models are commonly used for both retrospective and prospective analysis of the

* Corresponding author. Tel.: +1-607-255-1368; fax: +1607-255-8837

E-mail address: evan.cooch@cornell.edu (E.G. Cooch). dynamics of structured populations (Caswell, 2000, 2001), and are typically parameterized based on the transition rates among age- or size-classes 
assessed at some consistent point during the annual cycle. The choice of the actual timing of the annual census in the field (typically either prior to or immediately after breeding) is often determined by logistical constraints. In many cases, field studies focus on post-breeding census where enumerating the number of individuals in a population is made easier by the decreased mobility of individuals with offspring.

For birth-pulse populations enumerated immediately following breeding, survival for individuals age $i$ years is calculated as $l(i) / l(i-1)$, whereas for a pre-breeding census, survival is calculated as $l(i+1) / l(i)$, where $l(i)$ is the probability of surviving from birth to age $i$. Post-breeding fertility for individuals age $i$ years in birth-pulse populations is calculated as $\mathrm{S}_{i} \mathrm{~B}_{i}$, where $\mathrm{S}_{i}$ is the probability of survival from $t$ to $t+1$ (or, from age $i$ to age $i+1$; age and time are equivalent within a cohort). In contrast, fertility is calculated for a pre-breeding census as $\mathrm{B}_{i} l(1)=\mathrm{B}_{i} \mathrm{~S}_{0}$, the product of the expected fecundity (offspring production) of $i$ th age class $\left(\mathrm{B}_{i}\right)$ and survival from birth to just before first birthday $\left(\mathrm{S}_{0}\right)$.

Since survival and fertility estimates in either case are functions of common life-table parameters, it is straightforward to transform a postbreeding census model into a pre-breeding census model, and vice versa. In some cases, either a preor post-breeding matrix model may be preferred since it may provide a more useful (or at least convenient) separation of the individual sub-components of the $a_{i j}$ matrix elements for some analyses. For example, consider the following life-cycle diagram reflecting a post-breeding census of a population with 3 age-classes (offspring, 1year old and $2^{+}$-year old individuals; nodes 1,2 and 3 , respectively), with age-specific reproduction of both 2-year old $\left(\mathrm{B}_{S}\right)$ and $3^{+}$-year old $\left(\mathrm{B}_{A}\right)$ individuals, and juvenile (first-year) and adult survival $\left(\mathrm{S}_{J}\right.$ and $\mathrm{S}_{A}$, respectively).

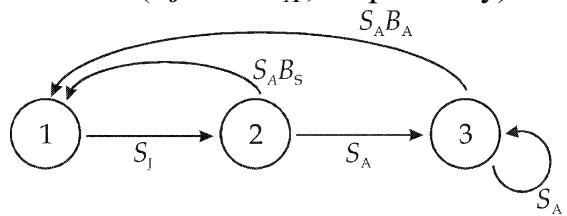

The projection matrix $\mathbf{A}^{\text {post }}$ corresponding to this life-cycle diagram is
$\mathbf{A}^{\text {post }}=\left(\begin{array}{ccc}0 & \mathrm{~S}_{A} \mathrm{~B}_{S} & \mathrm{~S}_{A} \mathrm{~B}_{A} \\ \mathrm{~S}_{J} & 0 & 0 \\ 0 & \mathrm{~S}_{A} & \mathrm{~S}_{A}\end{array}\right)$

The fertility elements ( $a_{1,2}$ and $a_{1,3}$ in this example) in a model based on a post-breeding census are functions of both adult survival $\left(\mathrm{S}_{A}\right)$ and offspring production $\left(\mathrm{B}_{x}\right)$.

However, it is possible to separate these two parameters by simply restructuring the model assuming a pre-breeding census. Since fertility in a pre-breeding census model is given as $\mathrm{B}_{i} l(1)=$ $\mathrm{B}_{i} \mathrm{~S}_{0}$, then for the present example, the life-cycle diagram corresponding to a pre-breeding census is

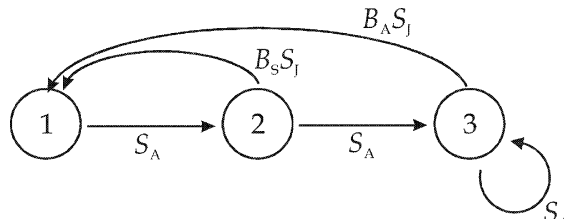

where nodes 1, 2 and 3 now refer to 1-year old, 2year old and 3-year old individuals, respectively. The projection matrix $\mathbf{A}^{\text {pre }}$ corresponding to this pre-breeding life-cycle diagram is

$\mathbf{A}^{\mathrm{pre}}=\left(\begin{array}{ccc}0 & \mathrm{~B}_{S} \mathrm{~S}_{J} & \mathrm{~B}_{S} \mathrm{~S}_{J} \\ \mathrm{~S}_{A} & 0 & 0 \\ 0 & \mathrm{~S}_{A} & \mathrm{~S}_{A}\end{array}\right)$

With a pre-breeding census, adult survival and reproductive output are separated.

Despite the structural changes, however, the projected asymptotic growth rate (given as the dominant eigenvalue $\lambda$ of the matrix; Caswell, 2001) is identical in both the pre- and postbreeding configurations (i.e., $\lambda_{\text {post }}=\lambda_{\text {pre }}$ ). This identity arises because annual survival (the probability of surviving a given annual interval) is (by definition) invariant in the annual cycle when it is estimated (i.e., winter to winter annual survival must be the same as summer to summer annual survival). Thus, estimated annual survival (or, products of survival with other transition rates estimated over the entire year) can be used in any matrix model that projects over the annual cycle, regardless of the timing of the census.

Given the invariance in growth rate for a single, deterministic projection model to the timing of the 
census, it might seem reasonable to assume that the long-term expected stochastic growth rate should not depend on whether the matrices are configured based on either a pre- or post-breeding census (Caswell, 2001). Recently, however, one of us (Gauthier) observed a case where the stochastic grow rates for models of the dynamics of the greater snow goose (Chen caerulescens atlantica) appeared to differ depending on whether or not a pre- or post-breeding model was used. Since this seemed to be inconsistent with theory (Caswell, 2001), we proceeded to explore the problem more fully. We show that such a discrepancy is, in fact, an artifact of the way that variation was introduced into our stochastic simulations. We explain the conditions under which such a discrepancy in stochastic growth rate between pre- and postbreeding census models can arise, and describe a somewhat general solution using seasonal (periodic) matrices.

\subsection{The apparent conundrum: a worked example}

Consider the following situation, based on a hypothetical goose population. For purposes of this example, the dynamics of the populations are completely specified by juvenile and adult survival $\left(\mathrm{S}_{J}\right.$ and $\mathrm{S}_{A}$, respectively), and basic reproductive output (B). Typical for goose populations, we assume that basal reproductive output $\mathrm{B}_{x}$ increases with age $x$, from $x=2$ to 4 years, beyond which there is no further change with age (Cooke et al., 1995). Based on a post-breeding census, the corresponding life cycle diagram and projection matrix $\left(\mathbf{A}^{\text {post }}\right)$ would be

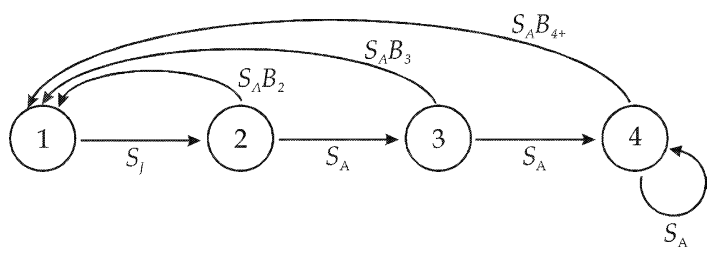

$$
\mathbf{A}^{\text {post }}=\left(\begin{array}{cccc}
0 & \mathrm{~S}_{A} \mathrm{~B}_{2} & \mathrm{~S}_{A} \mathrm{~B}_{3} & \mathrm{~S}_{A} \mathrm{~B}_{4+} \\
\mathrm{S}_{J} & 0 & 0 & 0 \\
0 & \mathrm{~S}_{A} & 0 & 0 \\
0 & 0 & \mathrm{~S}_{A} & \mathrm{~S}_{A}
\end{array}\right)
$$

while for a pre-breeding census, the projection matrix $\mathbf{A}^{\text {pre }}$ is

$\mathbf{A}^{\mathrm{pre}}=\left(\begin{array}{cccc}0 & \mathrm{~B}_{2} \mathrm{~S}_{J} & \mathrm{~B}_{3} \mathrm{~S}_{J} & \mathrm{~B}_{4+} \mathrm{S}_{J} \\ \mathrm{~S}_{A} & 0 & 0 & 0 \\ 0 & \mathrm{~S}_{A} & 0 & 0 \\ 0 & 0 & \mathrm{~S}_{A} & \mathrm{~S}_{A}\end{array}\right)$

We linked environmental stochasticity to our matrix models by creating a fixed set of $\mathrm{n}$ matrices $\left\{\mathbf{A}_{1}, \mathbf{A}_{2}, \ldots, \mathbf{A}_{n}\right\}$, where each matrix $\mathbf{A}_{i}$ corresponds to a given environmental state $i$. The longterm expected stochastic growth rate is then calculated by the product of matrices selected according to some random distribution from this set (Tuljapurkar and Orzack, 1980; Bierzychudek, 1982; Caswell, 2001). Other approaches to adding stochasticity to matrix models are noted in the discussion.

For this example, we assumed 2 environmental states, which we designate as either 'good' (g) or 'poor' (p). We used the following hypothetical data, corresponding to the parameters $\mathrm{B}_{x}, \mathrm{~S}_{J}$ and $\mathrm{S}_{A}$ for a 'typical' goose population in a 'good' and 'poor' year.

\begin{tabular}{lll}
\hline Parameter & 'good year' & 'poor year' \\
\hline $\mathrm{B}_{2}$ & 1.1 & 0.8 \\
$\mathrm{~B}_{3}$ & 1.5 & 1.2 \\
$\mathrm{~B}_{4+}$ & 1.9 & 1.5 \\
$\mathrm{~S}_{\mathrm{J}}$ & 0.45 & 0.25 \\
$\mathrm{~S}_{\mathrm{A}}$ & 0.75 & 0.75 \\
\hline
\end{tabular}

We varied both basal reproduction $\mathrm{B}$ and juvenile survival $\mathrm{S}_{J}$ between good and poor years, reflecting our general observation that, in most goose populations, there is considerable annual variation in basal reproduction and juvenile survival, but not adult survival (Cooch and Cooke, 1991; Francis et al., 1992; Cooch et al., 2001; Gauthier et al., 2001; Menu et al., 2002). This is consistent with the high elasticity for adult survival calculated for geese and most similar long-lived species (Rockwell et al., 1997; Gauthier and Brault, 1998; Pfister, 1998; Gaillard et al., 2000). Given these parameter values, the corresponding post- and pre-breeding census matrices for both 'good' and 'poor' years are 


$$
\begin{aligned}
\mathbf{A}_{g}^{\text {post }} & =\left(\begin{array}{cccc}
0 & 0.825 & 1.125 & 1.425 \\
0.450 & 0 & 0 & 0 \\
0 & 0.750 & 0 & 0 \\
0 & 0 & 0.750 & 0.750
\end{array}\right) \\
\mathbf{A}_{p}^{\text {post }} & =\left(\begin{array}{cccc}
0 & 0.600 & 0.900 & 1.125 \\
0.250 & 0 & 0 & 0 \\
0 & 0.750 & 0 & 0 \\
0 & 0 & 0.750 & 0.750
\end{array}\right) \\
\mathbf{A}_{g}^{\text {pre }} & =\left(\begin{array}{cccc}
0 & 0.495 & 0.675 & 0.855 \\
0.750 & 0 & 0 & 0 \\
0 & 0.750 & 0 & 0 \\
0 & 0 & 0.750 & 0.750
\end{array}\right) \\
\mathbf{A}_{p}^{\text {pre }} & =\left(\begin{array}{cccc}
0 & 0.200 & 0.300 & 0.375 \\
0.750 & 0 & 0 & 0 \\
0 & 0.750 & 0 & 0 \\
0 & 0 & 0.750 & 0.750
\end{array}\right)
\end{aligned}
$$

As expected, the projected deterministic growth rates for 'good' or 'poor' matrices are the same for either the post-breeding $\left(\lambda_{g}^{\text {post }}=1.182, \quad \lambda_{b}^{\text {post }}=\right.$ $0.991)$ or pre-breeding models $\left(\lambda_{g}^{\text {pre }}=1.182\right.$, $\left.\lambda_{b}^{\text {pre }}=0.991\right)$.

However, significant differences in the stochastic growth rate between the two configurations were observed, depending upon whether or not we used a pre- or post-breeding configuration. We estimated stochastic growth rate $\left(\log \hat{\lambda}_{s}\right)$ for these hypothetical data using the MLE described by Heyde and Cohen (1985). (Note: we adopt the $\log \hat{\lambda}_{s}$ notation (sensu Caswell, 2001); $\log \hat{\lambda}_{s}$ is equivalent to the $\log \lambda$ (Cohen, 1979) and $a$ (Tuljapurkar, 1990) notation used elsewhere.) We assumed that years could be classified as being either 'good' or 'poor', with $P($ 'good' $)=$ $P($ 'poor' $)=0.5$, selected iid. We projected 10000 generations for each of 1000 iterations, estimating growth as the mean of the $\log \left(\mathbf{N}_{t+1} / \mathbf{N}_{t}\right)$ (which is equivalent to the Heyde and Cohen MLE; Caswell, 2001), after discarding the first 500 generations for each iteration to eliminate the possible effect of transients. Population size was normalized after each generation (by dividing the population vector by the sum of the vector) to prevent cumulative errors in numerical precision. Although Heyde and Cohen (1985) described an analytical estimate for the variance of the stochastic growth rate, we derived $95 \%$ CI for $\log \hat{\lambda}_{s}$ numerically by using the 2.5 and $97.5 \%$ tails from the distribution of 1000 iterations. Using our hypothetical 'goose' population models, the estimated stochastic growth rate for the postbreeding census models was $\log \hat{\lambda}_{s}^{\text {post }}=0.0791$ (95\% CI: 0.0754-0.0824), while the estimated stochastic growth rate for the pre-breeding census models was $\log \hat{\lambda}_{s}^{\text {pre }}=0.0867 \quad(95 \%$ CI: $0.0830-$ $0.0903)$. These estimates are significantly different given a nominal Type I error rate of $\alpha=0.05$, with the pre-breeding models being projected to show greater stochastic growth than post-breeding models. Taken at face value, there would imply a considerable potential for error in estimating the stochastic growth rate of the population, simply as a function of how the apparent timing of the census upon which the model is parameterized.

\section{Conundrum explained: the problem of variable environmental states}

This result was surprising, since we had assumed that growth rate in general (stochastic or otherwise) was invariant to when in the annual cycle the population was censused (Caswell, 2001). However, we noted that if we varied only one parameter (say, juvenile survival, $\mathrm{S}_{J}$ ) between good and poor years, rather than simultaneously varying both juvenile survival and basal reproduction (as above), then no difference between $\hat{\lambda}_{s}^{\text {post }}$ and $\hat{\lambda}_{s}^{\text {pre }}$ was observed. For example, if we set basal reproduction $\mathrm{B}_{x}$ to be the same between good and poor years, using the previous values for good years in both cases (i.e., $\mathrm{B}_{2}=1.1, \mathrm{~B}_{3}=1.5$, and $\mathrm{B}_{4+}=1.9$ ), but used $\mathrm{S}_{J}=0.25$ for poor years, and $\mathrm{S}_{J}=0.45$ for good years, we estimated $\log \hat{\lambda}_{s}^{\text {post }}=$ $0.1067(95 \%$ CI: $0.1041-0.1092)$ and $\log \hat{\lambda}_{s}^{\text {pre }}=$ 0.1067 (95\% CI: 0.1037-0.1094). In this case, there is virtually complete overlap of the $95 \% \mathrm{CI}$, indicating no difference whatsoever in the estimated stochastic growth rates.

Careful examination of this subtle difference (aided by suggestions from Shripad Tuljapurkar and Stephen Ellner), yielded the explanation: simply restructuring the matrices between preand post-breeding configurations (as we had done) does not necessarily adequately account 


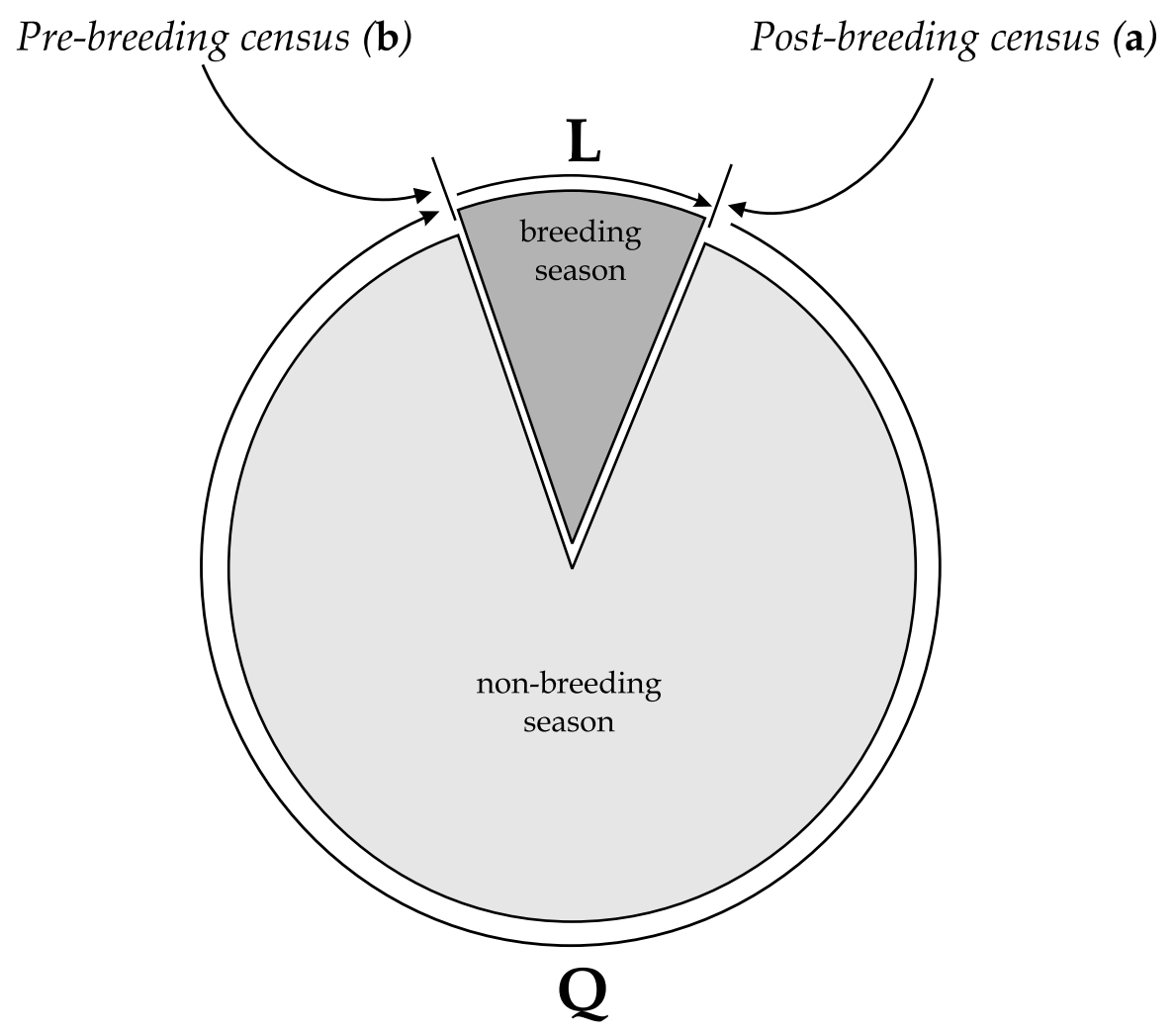

Fig. 1. Schematic representation of the annual cycle of a typical species of Arctic-breeding goose, partitioned into a breeding and nonbreeding season (size of the respective sections is drawn approximately proportional to the segment of the year comprised by each period). Populations can be censused either prior to (before) breeding (b) or after breeding (a). The transitions between the population vectors $\mathbf{a}$ and $\mathbf{b}$ are a function of the seasonal transition matrices $\mathbf{L}$ (breeding period) and $\mathbf{Q}$ (post- or non-breeding season), respectively.

for the time frame over which variation in the system occurs. As long as variation occurs within the same logical time period (say, calendar year), then both pre- and post-breeding models yield the same stochastic growth rates. However, if the pattern of variation occurs over more than one time frame (as is the case when more than one variable is changed between good and poor years), then failure to account for this difference leads to apparent differences in stochastic growth.

\subsection{A seasonal matrix approach}

This is most easily demonstrated using a periodic (seasonal) matrix approach (Caswell, 2001). We consider an annual cycle divided into 2 discrete phases, a breeding period and a non-breeding period (Fig. 1). We assume that the population can be censused either immediately prior to or immediately following breeding (referred to as above as pre- or before-breeding and post- or after-breeding, respectively). Let $\mathbf{b}(\mathbf{t})$ be the population vector before breeding in year $(t)$. Let $\mathbf{a}(\mathbf{t})$ be the population vector after breeding in year $(t)$. Let $\mathbf{L}(\mathbf{t})$ represent the transition matrix from $\mathbf{b}(\mathbf{t})$ to $\mathbf{a}(\mathbf{t})$, such that $\mathbf{a}(\mathbf{t})=\mathbf{L}(\mathbf{t}) \mathbf{b}(\mathbf{t})$ (i.e., $\mathbf{L}(\mathbf{t})$ is the breeding season transition matrix, incorporating breeding-season survival and fertility of individuals in the population vector $\mathbf{b}(\mathbf{t})$; Fig. 1). Let $\mathbf{Q}(\mathbf{t})$ be the transition matrix from $\mathbf{a}(\mathbf{t})$ to $\mathbf{b}(\mathbf{t}+\mathbf{1})$, such that $\mathbf{b}(\mathbf{t}+\mathbf{1})=\mathbf{Q}(\mathbf{t}) \mathbf{a}(\mathbf{t})$ (i.e., $\mathbf{Q}(\mathbf{t})$ is the nonbreeding season transition matrix, incorporating non-breeding season survival of individuals in the population vector a(t); Fig. 1). 

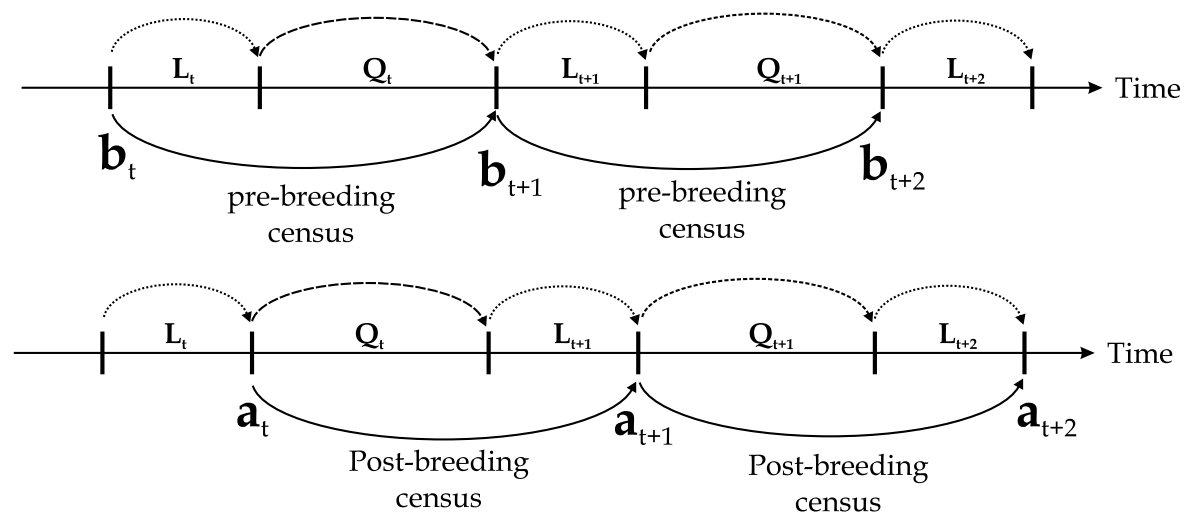

Fig. 2. Schematic representation of the time frames for each seasonal matrix $\mathbf{Q}$ and $\mathbf{L}$ (dashed arcs), and the annual projection matrix (solid arcs) corresponding to a pre- or post-breeding census model. $\mathbf{b}_{i}$ and $\mathbf{a}_{i}$ give the pre- and post-breeding population vectors in a given year, respectively.

Thus, $\quad \mathbf{b}(\mathbf{t}+\mathbf{1})=\mathbf{Q}(\mathbf{t}) \mathbf{a}(\mathbf{t})=\mathbf{Q}(\mathbf{t}) \mathbf{L}(\mathbf{t}) \mathbf{b}(\mathbf{t}), \quad$ while $\mathbf{a}(\mathbf{t}+\mathbf{1})=\mathbf{L}(\mathbf{t}+\mathbf{1}) \mathbf{b}(\mathbf{t}+\mathbf{1})=\mathbf{L}(\mathbf{t}+\mathbf{1}) \mathbf{Q}(\mathbf{t}) \mathbf{a}(\mathbf{t})$. Thus, the pre-breeding matrix can be written as $\mathbf{Q}(\mathbf{t}) \mathbf{L}(\mathbf{t})$, whereas the post-breeding census is $\mathbf{L}(\mathbf{t}+\mathbf{1}) \mathbf{Q}(\mathbf{t})$ (Fig. 2). Stated simply, the size of the pre-breeding population next year $(t+1)$ is a function of the size of the pre-breeding population this year $(t)$, successively multiplied by the breeding season and non-breeding season transition matrices $\mathbf{L}$ and $\mathbf{Q}$ for year $(t)$. In contrast, the size of the post-breeding population next year $(t+$ 1 ) is a function of the size of the post-breeding

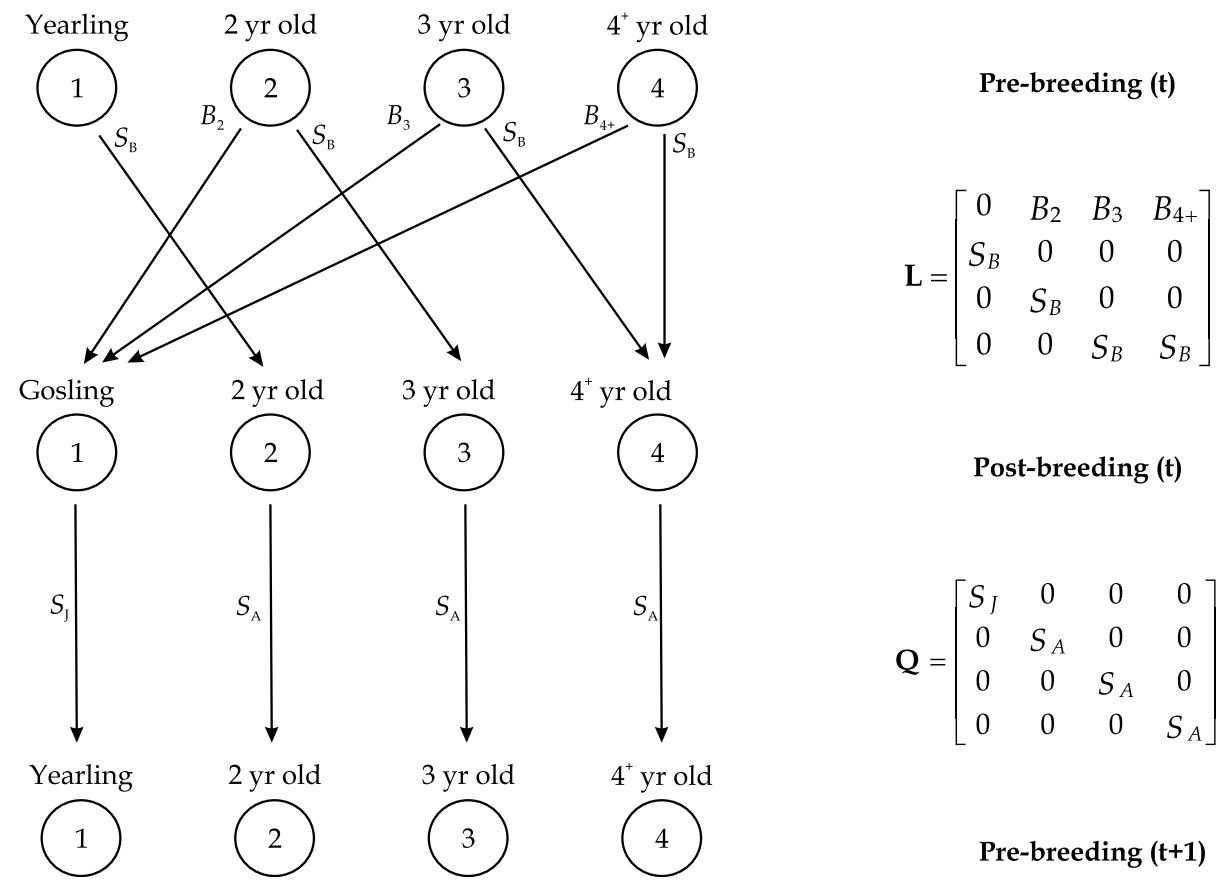

Fig. 3. A life cycle graph for the seasonal matrices corresponding to the breeding period (L) and post- or non-breeding period $(\mathbf{Q})$ for a hypothetical goose population (Fig. 1). The graph begins with a pre-breeding census in year $t$. For convenience, the starting phase is repeated at the bottom of the graph. 
population this year $(t)$, successively multiplied by the non-breeding season transition matrix $\mathbf{Q}$ for year $(t)$, and the breeding season transition matrix $\mathbf{L}$ for next year $(t+1)$ (Fig. 2).

In our modeling, we made several assumptions. First, we assumed that (1) years can be classified as being either 'good' or 'poor'; (2) that either $\mathbf{Q}$, or $\mathbf{L}$, or both, vary between good and poor years; and (3) in a given year $(t)$, if the year is categorized as good or poor, then this is reflected in both $\mathbf{L}$ and $\mathbf{Q}$ for that year (i.e., a good breeding season in year $(t)$ is followed by a good non-breeding season in year $(t))$. This third assumption is central to the rest of the argument. If either of the seasonal matrices $\mathbf{Q}$ or $\mathbf{L}$ is fixed (i.e., is constant over good and bad years), while the other varies, then the pre- and post-breeding census models both have 2 possible environmental states. For example, if $\mathbf{Q}$ is fixed, but $\mathbf{L}$ varies between good and bad years, then the 2 possible pre-breeding environmental states are $\mathbf{Q L}(g)_{t}$ and $\mathbf{Q L}(p)_{t}$, while the 2 possible post-breeding environmental states are $\mathbf{L}(g)_{t} \mathbf{Q}$ and $\mathbf{L}(p)_{t} \mathbf{Q}$.

In contrast, if both components vary, such that $\mathbf{Q}$ has 2 environmental states, and $\mathbf{L}$ has 2 environmental states (corresponding to good and poor years, respectively, in each case), then the pre-breeding census still has only 2 environmental states (since $\mathbf{Q}$ and $\mathbf{L}$ are both indexed to the same year $(t))$, but the post-breeding census model, in which $\mathbf{Q}$ and $\mathbf{L}$ are indexed in different years (i.e., $\mathbf{L}(\mathbf{t}+\mathbf{1}) \mathbf{Q}(\mathbf{t}))$, has 4 possible states: $\mathbf{L}(g)_{t+1} \mathbf{Q}(p)_{t}$, $\mathbf{L}(p)_{t+1} \mathbf{Q}(p)_{t}, \mathbf{L}(g)_{t+1} \mathbf{Q}(g)_{t}$, and $\mathbf{L}(p)_{t+1} \mathbf{Q}(g)_{t}$.

This provides the explanation for the difference in estimated stochastic growth rate when both $\mathbf{Q}$ and $\mathbf{L}$ are allowed to vary (analogous to varying $\mathbf{B}$ and $\mathrm{S}_{J}$ simultaneously in our earlier example), but not when we varied only one parameter. In the case of varying 2 parameters, the pre-breeding models have only two possible environmental states, where both $\mathbf{Q}$ and $\mathbf{L}$ are indexed to the same year $(t)$. In contrast, the post-breeding model has 4 possible environmental states, in each of which $\mathbf{Q}$ and $\mathbf{L}$ are indexed to different years. Thus, in the pre-breeding models, year $(t)$ is truly independent of year $(t+1)$. However, the situation is more complex for the post-breeding model: since the post-breeding projection is $\mathbf{L}(\mathbf{t}+\mathbf{1}) \mathbf{Q}(\mathbf{t})$, then if
$\mathbf{L}(\mathbf{t}+\mathbf{1})$ is a 'good year', then $\mathbf{Q}(\mathbf{t}+\mathbf{1})$ is also a 'good' year, meaning that the projection for $(t+2)$ is not independent of what happens at $(t+1)$. In our initial approach to estimating stochastic growth, where we varied both $\mathrm{S}_{J}$ and $\mathrm{B}_{x}$ between good and poor years (analogous to varying both $\mathbf{Q}$ and $\mathbf{L}$, respectively), we implicitly considered only $2(g-g$, and $p-p)$ out of the 4 possible postbreeding matrices (representing the 4 possible environmental states noted earlier), because of assumption 3 (above). Further, in simply transforming a pre-breeding census model to a postbreeding census model, we ignored the different time-frames used for $\mathbf{L}$ and $\mathbf{Q}$ in the post-breeding models, and the lack of independence between years.

\subsection{Application: the goose example}

Applying this seasonal (periodic) matrix modeling approach to our hypothetical goose example (Fig. 3), where both basal reproduction $\mathrm{B}_{x}$ and juvenile survival $\mathrm{S}_{J}$ varied between good and poor years, yielded 4 discrete matrices, corresponding to the breeding $(\mathbf{L})$ and non-breeding $(\mathbf{Q})$ segments of the annual cycle in both good and poor years (note: we assumed that survival of adults during breeding was 1.0, as indicated on the diagonal of the $\mathbf{L}_{i}$ matrices):

$\begin{aligned} \mathbf{L}_{g} & =\left(\begin{array}{cccc}0 & 1.1 & 1.5 & 1.9 \\ 1 & 0 & 0 & 0 \\ 0 & 1 & 0 & 0 \\ 0 & 0 & 1 & 1\end{array}\right) \mathbf{Q}_{g}=\left(\begin{array}{cccc}0.45 & 0 & 0 & 0 \\ 0 & 0.75 & 0 & 0 \\ 0 & 0 & 0.75 & 0 \\ 0 & 0 & 0 & 0.75\end{array}\right) \\ \mathbf{L}_{p} & =\left(\begin{array}{cccc}0 & 0.8 & 1.2 & 1.5 \\ 1 & 0 & 0 & 0 \\ 0 & 1 & 0 & 0 \\ 0 & 0 & 1 & 1\end{array}\right) \mathbf{Q}_{p}=\left(\begin{array}{cccc}0.25 & 0 & 0 & 0 \\ 0 & 0.75 & 0 & 0 \\ 0 & 0 & 0.75 & 0 \\ 0 & 0 & 0 & 0.75\end{array}\right)\end{aligned}$

Remembering that the pre-breeding census projection is the product $\mathbf{Q}(\mathbf{t}) \mathbf{L}(\mathbf{t})$, while the postbreeding census projection is the product $\mathbf{L}(\mathbf{t}+$ 1)Q(t), then we see clearly that the stochastic projection for the pre-breeding model involves selecting both $\mathbf{Q}$ and $\mathbf{L}$ for the same environmental state in each year $t$ (i.e., both good or both poor), while for the post-breeding model, we select $\mathbf{Q}$ for year $t$, but $\mathbf{L}$ for year $(t+1)$. This is easily accomplished computationally by initially constructing a vector containing the random series 
of environmental states, and then selecting the $(t+$ 1)th element to specify the environmental state for $\mathbf{L}$ given the environmental state for $\mathbf{Q}$ in year $(t)$. This also allows us to easily specify that $\mathbf{Q}(\mathbf{t}+\mathbf{1})$ in the next iteration must reflect the same environmental state as for $\mathbf{L}(\mathbf{t}+\mathbf{1})$, under the assumption that $\mathbf{Q}$ and $\mathbf{L}$ both reflect the same environmental state within any annual period. Using this seasonal matrix approach, the estimated stochastic growth rate for the pre-breeding census models $\log \hat{\lambda}_{s}^{\text {pre }}=$ 0.0867 (95\% CI: $0.0833-0.0900$ ), while the estimated stochastic growth rate for the post-breeding census models was $\log \hat{\lambda}_{s}^{\text {post }}=0.0868(95 \% \mathrm{CI}$ : $0.0833-0.0902)$. Clearly, there is now no difference in the estimated stochastic growth rate between the two configurations. We note that the estimate of $\log \hat{\lambda}_{s}^{\text {pre }}\left(=\log \hat{\lambda}_{s}^{\text {post }}\right)=0.0867$ is identical with the estimate from our original calculation for the pre-breeding model (presented earlier), as is the 95\% CI (showing the equivalence of the two approaches in the pre-breeding case). Not surprisingly, we found similar equivalence when we varied only one parameter at time.

\section{Discussion}

This short commentary is intended to reinforce the general suggestion that, whenever dealing with stochastic systems in discrete time, care must be taken to ensure that the manner in which stochasticity is entered into the system actually reflects what the investigators intend. We were unwittingly guilty of not heeding this general admonishment, and found ourselves faced with what appeared to be an incongruous result: in some cases, we seemed to have a statistically significant difference in estimated stochastic growth rates depending on whether or not the matrix models were parameterized assuming either a pre- or post-breeding census. However, such a difference seems to contradict basic expectations from standard ergodic theory (Caswell, 2001).

Fortunately, there is a relatively straightforward explanation for the differences that we observed: such differences can occur if there is (i) a difference in the number of possible states between the preand post-breeding configurations, and (ii) care is not taken to properly time-subscript the seasonal matrices ( $\mathbf{Q}$ and $\mathbf{L}$ in our examples) which when multiplied together yield the annual projection matrix A. Using a periodic (seasonal) matrix approach, with explicit subscripting, avoids this problem, and eliminated the apparent differences between pre- and post-breeding results which occurred in some cases.

However, there are at least two important points to make here. First, the 'problem' occurred when considering post-breeding census projections. For post-breeding census models, the projection is $\mathbf{L}(\mathbf{t}+\mathbf{1}) \mathbf{Q}(\mathbf{t})$. Thus, for post-breeding models, which are probably the most commonly used, it is critical to properly account for the timing of the 2 seasonal matrices $\mathbf{L}$ and $\mathbf{Q}$ : since the census occurs at the end of breeding in year $(t)$, then the projection involves the non-breeding season survival (Q) in year $(t)$, but the breeding season production matrix $\mathbf{L}$ in year $(t+1)$ ! Simply transforming a pre-breeding model into a post-breeding model by (in effect) changing where $\mathrm{S}_{A}$ and $\mathrm{S}_{J}$ occur in the matrix ignores this difference in time subscripting. And, as such, the estimated stochastic growth rate will differ from the estimate from a periodic matrix projection which properly subscripts the seasonal matrices.

Second, the pattern of covariance between $\mathbf{Q}$ and $\mathbf{L}$ may be important in some cases. As noted earlier, in our example we considered a particular 'calendar year' as being either 'good' or 'poor'. Thus, we assume that both $\mathbf{Q}$ and $\mathbf{L}$ are simultaneously classified as good or poor, depending on the year. For example, consider a post-breeding census model. Suppose that non-breeding survival matrix $\mathbf{Q}$ reflects a 'poor' year in year $(t)$, while the breeding period matrix $\mathbf{L}$ in year $(t+1)$ is a 'good' year. Then, this means that the non-breeding matrix $\mathbf{Q}$ next year must also reflect a 'good' year. This lack of independence, and failing to properly time-subscript the transition matrices, led to our original observation. This 'positive covariance' between $\mathbf{L}$ and $\mathbf{Q}$ is clearly not always the case, although it may in fact be generally correct for most goose populations (poor breeding years often are characterized by slow offspring growth and survival over the first year, such that $\mathbf{L}$ and $\mathbf{Q}$ covary positively within a year) and many other 
organisms. It is important to note that our original observation of a difference in the stochastic growth rates as a function of the timing of the census was a function of our failure to account for the covariance of environmental states, by properly accounting for the time-subscripting of $\mathbf{Q}$ and L (Fig. 2). (The same is true regardless of whether the covariance is positive, as in our case, or negative, although negative covariance in environmental states will clearly change the projected stochastic growth rates; in our example, $\log \hat{\lambda}_{s}=$ 0.0868 for positive environmental covariance, and $\log \hat{\lambda}_{s}=0.0746$ for negative environmental covariance). If $\mathbf{Q}$ and $\mathbf{L}$ vary independently within and among years, then the 'problem', as we have described it, disappears.

Our results are similar in several respects to consideration of stochastic growth rates in periodic systems, where it is often necessary to treat environmental variation as a Markov process, rather than iid (sensu Silva et al., 1991). As noted by Caswell (2001; p. 421) in some cases the frequency of a particular environmental state is insufficient to characterize the population growth rate (which it is in completely iid situations), because the order in which the environmental states occur is also important (see also Tuljapurkar, 1997). Markov chain models include both iid (no serial autocorrelation among environments) and periodic environments (serial autocorrelation approaching 1) as limiting cases (Dixon et al., 1997; Caswell, 2001). Failure to account for autocorrelation among environments can significantly influence both estimates of stochastic growth rates (Dixon et al., 1997; Tuljapurkar, 1997; Caswell, 2001), and the relative sensitivity of population growth to perturbation of one or more elements of the model (Dixon et al., 1997; but see Caswell 2001, p. 421).

\subsection{Entering stochasticity in matrix models}

The results of our analysis of this problem invariably leads to the general question of how best to introduce stochasticity into matrix models; as stochastic matrix models are increasingly used, such a consideration is important. As noted by Caswell (2001), there are a number of ways in which matrix models can be linked to stochastic environmental variation. One approach is to allow the elements of the matrices to vary independently, according to some parametric distribution (e.g., Maguire et al., 1996; Ratner et al., 1997). If each matrix element varies independently of the other elements of the matrix, then no difference in stochastic growth rate between pre- or postbreeding census models is observed. However, such an approach ignores likely covariance among matrix elements. Covariance among matrix elements can be achieved either by sampling the individual $a_{i j}$ values from the joint probability distribution (which will be multivariate, although generally not multivariate normal), or by randomly generating the lower-level vital rates from which the matrix entries are calculated (Doak et al., 1994; Caswell, 2001). While the former approach can be technically difficult (especially when the joint probability distribution is not multivariate normal), the latter is readily implemented. The difficulty with using randomly generated lower-level vital rates is in adequately specifying (i) the appropriate parametric distribution for the particular vital rate (the shape of the random distribution used can strongly influence the results of stochastic projections in some cases; Bukowski et al., 1995; Hamed and Bedient, 1997; Menges, 2000), and (ii) specifying the appropriate variance term for each parameter (which requires decomposing estimated variance into process and sampling variance; this is also not easily done in some instances, especially for parameters which are not estimated based on a formal statistical model; Burnham et al., 1987).

An alternative, easily implemented approach is to select a matrix from a set of matrices, where each matrix in the set corresponds to a particular environment. Since the matrix reflects the covariance among the matrix elements, then this in effect imposes a particular covariance structure on the vital rates. This approach has been widely used (Gauthier and Brault, 1998; Caswell and Kaye, 2000; Caswell, 2001). However, this approach selects from a finite set of matrices, each corresponding to a particular environmental state, and is in effect a bootstrap sample of possible environmental states. As such, inference is conditional on 
the adequacy of the bootstrap sample in representing the range of environmental states; with a finite number of matrices, there are a finite number of pathways (i.e., stochastic realizations) that a population can follow. Clearly, this is not a limitation if matrix elements are selected at random from parametric distributions.

\section{Conclusions}

This study was motivated by a result which made little sense to us: an observation that, under some conditions, the stochastic growth rate estimated from a matrix model based on a prebreeding census differed significantly from the growth rate estimated for the same model, but parameterized to reflect a post-breeding census. It is fair to note that such a comparison is somewhat artificial, and that the original result was conditional upon how we structured the model (specifically, the positive covariance between seasons; a good breeding season is followed by a good nonbreeding season). Regardless, our results demonstrate the need for careful thought as to how randomness is introduced into structured models, particularly when there is covariance among seasons (as there may often be). If care is not taken, particular with post-breeding census models, resulting estimates of stochastic growth rate may be biased. Seasonal (periodic) matrix models, as we have used, have the utility of making the pattern of stochasticity, and covariance among seasons, explicit. We reiterate the basic recommendation to explore stochastic matrix models (Caswell, 2001), but to do so carefully.

\section{Acknowledgements}

We gratefully acknowledge the consideration of our problem by Shripad Tuljapurkar (Stanford University) and Stephen Ellner (Cornell University), who made several 'leading' suggestions which helped us understand the solution to the problem, while graciously avoiding the use of the word 'obvious' (which it probably was to them). We also thank Jim Nichols for his careful review of the paper. Additional comments from Brett Sandercock (Kansas State University) improved the presentation.

\section{References}

Bierzychudek, P., 1982. The demography of jack-in-the pulpit, a forest perennial that changes sex. Ecol. Monogr. 52, 335351.

Bukowski, J., Korn, L., Wartenberg, D., 1995. Cirrelated inputs in quantitative risk assessment: the effects of distributional shape. Risk Anal. 15, 215-219.

Burnham, K.P., Anderson, D.R., White, G.C., Brownie, C., Pollock, K.H., 1987. Design and Analysis Methods for Fish Survival Experiments Based on Release-Recapture. American Fisheries Society, Bethesda, Maryland, p. 437.

Caswell, H., 2000. Prospective and retrospective perturbation analyses: their roles in conservation biology. Ecology 81, 619-627.

Caswell, H., Kaye, T., 2000. Stochastic demography and conservation of Lomatium bradshawii in a dynamic fire regime. Adv. Ecol. Res. 31, 1-51.

Caswell, H., 2001. Matrix Population Models-Construction, Analysis, and Interpretation, Second ed.. Sinauer Associates, Sunderland, Massachusetts, p. 722.

Cohen, J.E., 1979. Comparative statics and stochastic dynamics of age-structured populations. Theor. Pop. Biol. 16, 159171.

Cooch, E.G., Cooke, F., 1991. Demographic changes in a snow goose population: biological and management implications. In: Perrins, C.M., Lebreton, J.-D., Hirons, G.J.M. (Eds.), Bird Population Studies. Oxford University Press, Oxford, pp. $168-189$.

Cooch, E.G., Rockwell, R.F., Brault, S., 2001. Retrospective analysis of demographic responses to environmental change: an example in the lesser snow goose. Ecol. Monogr. $71,377-400$.

Cooke, F., Rockwell, R.F., Lank, D.B., 1995. The Snow Geese of La Pérouse Bay: Natural Selection in the Wild. Oxford University Press, Oxford, p. 297.

Dixon, P., Friday, N., Ang, P., Heppell, S., Kshatriya, M., 1997. Sensitivity analysis of structured-population models for management and conservation. In: Tuljapurkar, S., Caswell, H. (Eds.), Structured-Population Models in Marine, Terrestrial, and Freshwater Systems. Chapman and Hall, New York, pp. 471-513.

Doak, D., Lareiva, P., Klepetka, B., 1994. Modeling population viability for the desert tortoise in the western Mojave Desert. Ecol. Appl. 4, 446-460.

Francis, C.M., Richards, M.H., Cooke, F., Rockwell, R.F., 1992. Long-term changes in survival rates of lesser snow geese. Ecology 73, 1346-1362.

Gaillard, J.-M., Festa-Bianchet, M., Yoccoz, N.G., Loison, A., Toïgo, C., 2000. Temporal variation in fitness components 
and population dynamics of large herbivores. Annu. Rev. Ecol. Syst. 31, 367-393.

Gauthier, G., Brault, S., 1998. Population model of the greater snow goose: projected impacts of reduction in survival on population growth rate. In: Batt, B.D.J. (ed.), The Greater Snow Goose: Report of the Arctic Goose Habitat Working Group, Arctic Goose Joint Venture Special Publication. US Fish and Wildlife Service, Washington, DC and Canadian Wildlife Service, Ottawa, pp. 65-80.

Gauthier, G., Pradel, R., Menu, S., Lebreton, J.-D., 2001. Seasonal survival of greater snow geese and effect of hunting under dependence in sighting probability. Ecology $82,3105-3119$.

Hamed, M.M., Bedient, P.B., 1997. On the effect of probability distributions of input variables in public health risk assessment. Risk Anal. 17, 97-105.

Heyde, C.C., Cohen, J.E., 1985. Confidence intervals for demographic projections based on products of random matrices. Theor. Pop. Biol. 27, 120-153.

Maguire, L.A., Wilhere, G.F., Dong, Q., 1996. Population viability analysis for red-cockaded woodpeckers in the Georgia Piedmont. J. Wildl. Manage. 59, 533-542.

Menges, E.S., 2000. Population viability analyses in plants: challenges and opportunities. Trends Ecol. Evol. 15, 51-56.

Menu, S., Gauthier, G., Reed, A., 2002. Changes in survival rates and population dynamics of greater snow geese over a 30-year period: implications for hunting regulations. J. Appl. Ecol. 39, 91-102.
Pfister, A.C., 1998. Patterns of variance in stage-structured populations: evolutionary predictions and ecological implications. Proc. Natl. Acad. Sci. 95, 213-218.

Ratner, S., Lande, R., Roper, B.B., 1997. Population viability analysis of spring Chinook salmon in the South Umpqua River, Oregon. Cons. Biol. 11, 879-889.

Rockwell, R.F., Cooch, E.G. and Brault, S., 1997. Dynamics of the mid-continent population of lesser snow geese-projected impacts of reductions in survival and fertility on population growth rates. In: Batt, B. (ed.) Arctic Ecosystems in Peril: Report of the Arctic Goose Habitat Working Group. Arctic Goose Joint Venture, Canadian Wildlife Service, Ottawa and US Fish and Wildlife Service, Washington, DC, pp. 73-100.

Silva, J.F., Raventos, J., Caswell, H., Trevisan, M.C., 1991. Population responses to fire in a tropical savanna grass Andropogon semiberbis: a matrix model approach. J. Ecol. 79, 345-356.

Tuljapurkar, S., 1990. Population Dynamics in Variable Environments. Springer, New York, p. 154.

Tuljapurkar, S., 1997. Stochastic matrix models. In: Tuljapurkar, S., Caswell, H. (Eds.), Structured-Population Models in Marine, Terrestrial, and Freshwater Systems. Chapman and Hall, New York, pp. 59-87.

Tuljapurkar, S., Orzack, S.H., 1980. Population dynamics in variable environments. I. Long-run growth rates and extinction. Theor. Pop. Biol. 18, 314-343. 